\title{
openheart Mortality and oral anticoagulants in the Food and Drug Administration Adverse Event Reporting System
}

\author{
Victor Serebruany, ${ }^{1,2}$ Vasily Cherepanov, ${ }^{2}$ Seth Fortmann, ${ }^{2}$ Moo Hyun $\mathrm{Kim}^{3}$
}

To cite: Serebruany V,

Cherepanov V, Fortmann S, et al. Mortality and oral anticoagulants in the Food and Drug Administration Adverse Event Reporting System. Open Heart 2017;4:e000629. doi:10.1136/ openhrt-2017-000629

Received 15 March 2017 Revised 13 April 2017 Accepted 25 April 2017

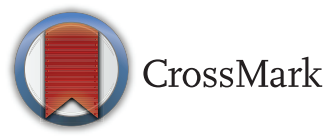

1 Johns Hopkins University, Baltimore, Maryland, USA ${ }^{2}$ HeartDrug ${ }^{\mathrm{TM}}$ Research Laboratories, Towson, Maryland, USA

${ }^{3}$ Dong-A University, Busan, South Korea

Correspondence to Moo Hyun Kim; kimmh@dau. ac.kr

\section{ABSTRACT}

Objective The comparative crude death rates (CDR) among non-vitamin $\mathrm{K}$ antagonist oral anticoagulants (NOACs) are unknown. Further, whether NOACs improve survival when compared with warfarin is also unclear. We compared CDR co-reported for four NOACs combined or separately versus warfarin within the US Food and Drug Administration (FDA) Adverse Event Reporting System (FAERS) database.

Methods We selected CDR from the FAERS database linked to four NOACs and warfarin. The primary endpoints were differences in proportional reporting ratios (PRRs), and Chi-Square $\left(\chi^{2}\right)$ for dabigatran, rivaroxaban, apixaban and edoxaban when compared with warfarin.

Results The FAERS database contains significantly less death reports associated with all NOACs combined (14917 out of 128267 reports $(11.63 \%)$; PRR=1.089; $\chi^{2}=70.0 ; p=6.05 \mathrm{e}^{-17}$ ) than for warfarin (19493 out of 153911 reports $(12.67 \%))$. The numbers for rivaroxaban ( 6318 out of 64512 reports or $(9.79 \%)$; $P R R=1.293$; $\chi^{2}=359.4 ; p=3.72 e^{-80}$ ), apixaban (1693 out of 17789 reports $(9.52 \%) ; P R R=1.331 ; \chi^{2}=145.8 ; p=1.43 \mathrm{e}^{-33}$ ) and edoxaban (53 out of 755 reports $(7.02 \%)$; PRR $=1.804$; $\chi^{2}=21.18 ; p=4.18 \mathrm{e}^{-06}$ ) were favourable as compared with warfarin, while the numbers of fatalities co-reported with dabigatran (6989 out of 46250 reports $(15.11 \%)$; PRR=0.838; $\chi^{2}=185.2 ; \mathrm{p}=3.61 \mathrm{e}^{-42}$ ) were higher than for warfarin.

Conclusion Overall, based on these CDR, NOACs appear to be associated with a mortality benefit over warfarin. Among NOACs, we observed remarkably similar for factor Xa inhibitors (rivaroxiban, apixaban and edoxaban) but unfavourable signal for the direct thrombin inhibitor (dabigatran). However, these data are clearly not sufficient to change the prescription patterns.

\section{INTRODUCTION}

The optimal choice of anticoagulant for numerous indications including stroke prevention in atrial fibrillation represents an unsolved medical issue. The non-vitamin $\mathrm{K}$ antagonist oral anticoagulants (NOACs) dabigatran, rivaroxaban, apixaban and edoxaban have been tested in large-scale randomised clinical trials against warfarin, but not in any head-to-head comparison studies. Since mortality is the most important hard outcome measure,

\section{KEY QUESTIONS}

What is already known about this subject?

- Non-vitamin K antagonist oral anticoagulants (NOACs) represent the cornerstone therapy for stroke prevention in patients with atrial fibrillation.

- The comparative mortality risks over warfarin, and among four different NOACs are unclear since randomised evidence is limited or not existing.

- The Food and Drug Administration Adverse Event Reporting System (FAERS) is a passive surveillance repository that contains information on adverse events, while associated fatalities are mandatory to be reported by pharmaceutical manufacturers.

What does this study add?

- The comparative total FAERS crude death rates suggest that NOACs appear to be associated with a mortality benefit over warfarin.

- Among NOACs, we observed favourable trends for factor Xa inhibitors (rivaroxaban, apixaban and edoxaban) but unfavourable signal for the direct thrombin inhibitor (dabigatran).

How might this impact on clinical practice?

- Reasonable caution and awareness are warranted for patients receiving dabigatran until completion of the FAERS 2016 safety review. Although the data are consistent and large, these findings need to be interpreted with utmost caution.

these initial indication-seeking trials ${ }^{1-4}$ and their meta-analysis ${ }^{5}$ suggest a mild all-cause mortality reduction with the relative risk of $0.90(95 \%$ CI 0.85 to $0.95, \mathrm{p}<0.001)$ for NOACs against warfarin. When considering differences between the NOACs, trial data cannot be extrapolated due to trial heterogeneity, differences in baseline populations, variations in antecedent warfarin use and different drug discontinuation rates. ${ }^{6}$ Furthermore, a Food and Drug Administration (FDA)-generated analysis found up to double-digit rates of discontinuations and incomplete follow-up rates in Randomized Evaluation of LongTerm Anticoagulation Therapy (RE-LY) (21\% and 9\%), Rivaroxaban Once Daily Oral 
Direct Factor Xa Inhibition Comparedwith Vitamin K Antagonism for Prevention of Stroke and Embolism Trial in Atrial Fibrillation (ROCKET-AF) (28\% and 20\%), Apixaban for Reduction in Stroke and Other ThromboembolicEvents in Atrial Fibrillation (ARISTOTLE) (25\% and $15 \%$ ) and Effective Anticoagulation with Factor Xa Next Generation in AtrialFibrillation (ENGAGE) (34\% and $10 \%$ ), respectively, ${ }^{7}$ suggesting that some outcomes were missing downgrading the validity of the trial results. In light of these methodological challenges, evidence from large-scale uniformed government-run databases and repositories may be helpful to pick up possible adverse event signals in general and death in particular.

The US FDA Adverse Event Reporting System (FAERS, formerly AERS) is a database that contains information on adverse event and medication error reports submitted to the FDA. FAERS is a passive surveillance system that relies on voluntary reporting by healthcare professionals and consumers, as well as required mandatory reporting by pharmaceutical manufacturers. FAERS includes spontaneous reports from US sources; serious and unlabelled spontaneous reports from non-US sources; and serious, unlabelled and attributable postmarketing clinical trial reports from all sources. ${ }^{8}$ Data mining algorithms have been developed for the quantitative detection of signals from such a large database, where a signal means a statistical association between a drug and an adverse event or a drug-associated adverse event, including advanced statistics such as the proportional reporting ratio (PRR) with $\chi^{2}$ allowing to detect significant differences. ${ }^{9}$ FAERS data are publicly available as files containing raw data of individual case safety reports. ${ }^{10}$ There are different methodologies and recent advances using data mining to detect drug interactions with impact on patient outcomes including death. Importantly, the data mining of different pharmacovigilance sources, such as FAERS and electronic health records are surprisingly rarely used in the scientific biomedical literature and social media. This is probably due to limited public awareness that such repository is indeed free to use and easily assessable..$^{7-10}$ In the current work, we compared total crude death reports associated with all NOACs combined, as well as four NOACs separately, versus warfarin within the entire FAERS database.

\section{METHODS}

\section{Data source}

We retrieved the data from the FAERS database and conducted analyses of deaths records. We performed drug mapping and created a list of synonyms for five anticoagulants. All FAERS records were searched by terms 'warfarin', 'dabigatran', 'rivaroxaban', 'apixaban', 'edoxaban', 'Pradaxa', 'Xarelto', 'Eliquis', 'Savaysa', 'Lixiana', 'Brumolin', 'Athrombine k', 'Coumadin', 'Coumafen', 'Coumafene', 'Coumaphene', 'Coumarin', 'Coumefene', 'Dethmor', 'Dethnel', 'Dicusate', 'Kumader', 'Kumadu', 'Kumatox', 'Kypfarin', 'Latka 42', 'Maveran', 'Marcoumar' 'Panwarfin', 'Prothromadin', 'Ratorex', 'Rodafarin', 'Rosex', 'Solfarin', 'Vampirinip', 'Warfarat', 'Warfarina', 'Warfarine', 'Warfarinum', 'Zoocoumarin' and 'death'. We deliberately avoided event mapping since we were looking for the single outcome (death). In fact, patient outcome of death has not changed in any of the various iterations of the FAERS database over the years, requiring no extra mapping. Duplicate reports were dealt with by combining reports with identical case numbers. There still may be other duplicates that we missed, but there is no clear method for identifying these 'hidden' duplicates, and we assume that the proportion of duplicates for each anticoagulant is not meaningfully different than the others. We specifically handled the missing data. The only missing data that we have is if a case does not report an 'outcome'. If a case did not report an outcome then we categorised this case as 'not death'. To avoid bias, data mining and statistics were performed by independent researchers at FDAble (Glastonbury, Connecticut, USA; http://www.fdable.com), a for-profit group that specialises in FAERS database analyses.

\section{Patient involvement}

FAERS is a public freely accessible database, where the patient identity is protected by coding. Patients, service users or providers were not involved in the design of this study. The development of outcome measure (mortality) was not informed by patients' priorities, experience and preferences. No patients were involved in the recruitment to and conduct of the index study. We do not plan to disseminate study results specifically to FAERS participants.

\begin{tabular}{|c|c|c|c|c|c|c|}
\hline Drug & Total cases (n) & Deaths (n) & Deaths (\%) & $\chi^{2 *}$ & p Value* & PRR $(95 \% \mathrm{Cl})$ \\
\hline Dabigatran & 46250 & 6989 & 15.11 & 185.2 & $3.61 \mathrm{e}^{-42}$ & 0.838 (0.817 to 0.860$)$ \\
\hline Rivaroxaban & 64512 & 6318 & 9.79 & 359.4 & $3.72 \mathrm{e}^{-80}$ & 1.293 (1.259 to 1.328$)$ \\
\hline Apixaban & 17789 & 1693 & 9.52 & 145.8 & $1.43 \mathrm{e}^{-33}$ & 1.331 (1.269 to 1.395$)$ \\
\hline Edoxaban & 755 & 53 & 7.02 & 21.2 & $4.18 \mathrm{e}^{-06}$ & 1.804 (1.391 to 2.340$)$ \\
\hline All NOACs & 128267 & 14917 & 11.63 & 70.0 & $6.05 \mathrm{e}^{-17}$ & 1.089 (1.067 to 1.111$)$ \\
\hline Warfarin & 153911 & 19493 & 12.67 & NA & NA & 1.000 \\
\hline
\end{tabular}

*When compared with warfarin.

FAERS, Food and Drug Administration Adverse Event Reporting System; NA, not applicable; NOACs, non-vitamin K antagonist oral anticoagulants; PRR, proportional reporting ratio. 


\section{Outcomes}

The primary endpoint of this study was death rates co-reported with dabigatran, rivaroxaban, apixaban, edoxaban and compared with warfarin within FAERS. To mitigate the issue of multiple reporting of a single event, deaths were counted by unique case numbers rather than by report numbers. In other terms, if a single case has three separate reports and each report indicates 'death', the mandatory counting was a single death, and two other deaths reports were disregarded. The Medical Dictionary for Regulatory Activities (MedDRA) search was irrelevant, since we were using patient outcome (death), which is not covered by MedDRA.

\section{Statistical analysis}

Analyses were done using Open VigilFDA V.1.0.2, a web-based user interface for the FAERS database. ${ }^{89}$ This software allows for the analysis of adverse drug events reported to the FDA. These adverse events can then be analysed for 'disproportionality' and scored using various measures of statistical significance like PRR. ${ }^{8-10}$ These contemporary statistical techniques compare the reported adverse events to expected adverse events and allow quantifying the additional risk/odds of the drug and adverse event to the general background noise. Proportional reporting ratios (PRR) and reporting ORs (RORs) and $95 \%$ CIs were calculated as a measure of disproportionality of reporting, and RORs were compared across drugs with the Breslow-Day statistics. Values above 1 suggest a disproportionate association of a drug and event. Roughly, values greater than 2 indicate that this drug to adverse event combination is twice as likely as all other combinations. $\chi^{2}$ estimates with Yates correction were assessed for data validation purposes suggestive of whether there are enough entries for analysis to be

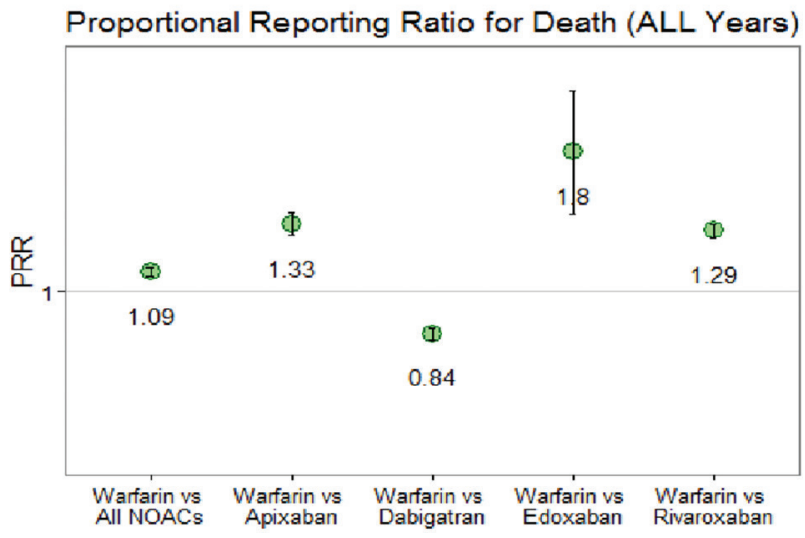

Figure 1 FAERS death cases (all years) for NOACs versus warfarin. Warfarin is arbitrated at PRR $=1.00$ and co-report death 1.33 times more frequently than with apixaban/ death, co-report death 1.29 times more frequently than with rivaroxaban/death, co-report death 1.80 times more frequently than with edoxaban/death, but co-report death 0.84 times less frequently than dabigatran/death. FAERS, Food and Drug Administration Adverse Event Reporting System; NOACs, non-vitamin K antagonist oral anticoagulants. meaningful and to assess the $\mathrm{p}$ values. ${ }^{11}$ The bigger the $\chi^{2}$ number, the more likely the two groups are different based on that feature.

\section{RESULTS}

From 7732656 screened FAERS records, we excluded 7449439 and qualified 283217 events including 34546 co-reported deaths. The retroactive event extraction matched the first data entry, that is, 2 November 1997 for warfarin, 15 March 2005 for dabigatran, 9 October 2007 for rivaroxaban, 21 September 2006 for apixaban and 21 May 2012 for edoxaban. The cut-off was 31 December 2015 for all five anticoagulants. Overall, the FAERS contains 248271 survival records and 34546 $(12.2 \%)$ fatalities associated with all NOACs and warfarin combined. If two different anticoagulants are reported in a single case, we count that case for both anticoagulants. This explains why the total number of cases for all NOACs (128 267) is slightly less than the sum of cases for dabigatran, rivaroxaban, apixaban and edoxaban (129 306). Understandably, records linked to warfarin were dominant representing over $60 \%$ of the entire dataset. Among NOACs, rivaroxaban reported the most events, followed by dabigatran, apixaban and very few $(<1000)$ edoxaban entries. The total distribution of combined and individual drug deaths co-reported in FAERS for the four NOACs and for warfarin is presented in table 1 , while the comparative PRR values are illustrated in figure 1. Relative PRR risks for deaths are exhibited in figure 1.

\section{DISCUSSION}

These large uniformed international repository data point to an overall mild superiority of NOACs over warfarin with regards to reduced mortality risks, with one exception. A disproportional excess of mortality signal associated with dabigatran was observed when compared with warfarin, and especially with the other three NOACs. To immediately focus on this latter finding, we do not know whether we are comparing apples with oranges when describing the individual NOAC populations, hence this result deserves extreme interpretative caution.

We deliberately focused our analyses exclusively on mortality for three major reasons. First, death is the most important outcome measure validating the efficacy of any drug in general, and NOACs in particular. There are certain FAERS algorithms which are proven to be effective at identifying prelinked duplicate reports. Importantly, the narrative text manual reviews are not particularly useful, but rather automated detection evaluation should be applied for proper counts. Second, the FAERS database analyses are always challenged by the mixture of patients and reports, since any single event can generate multiple reports. In contrast, death is a final outcome avoiding numerator confusion or/and repeated denominator counts. Third, despite the uniformed regulatory decisions to approve different NOACs at several doses for various indications, most reliable mortality data 
are currently based on a single trial results. The problem is that claiming superiority over other anticoagulants is tricky and should be judged with great caution considering the lack of definite evidence, especially with regards to comparative studies among NOACs. NOAC manufacturers are hesitant to conduct superiority studies limiting the evidence to the indication-seeking initial pivotal trials against warfarin.

Overall, it appears reassuring that NOACs are emerging with a death benefit over warfarin. These data are in agreement with the magnitude of such effect as reported in a pooled trial meta-analysis. However, we find it concerning that among NOACs, only dabigatran appears to be linked to a disproportionate signal of extra deaths in FAERS, while rivaroxaban, apixaban and edoxaban fare superior to warfarin with regard to associated mortality risks. Notably, the degree of death reduction with rivaroxaban, apixaban and edoxaban over warfarin look remarkably consistent in FAERS.

There are few important considerations to be brought in from the current dataset. First, the sample size of adverse event reports in general, and deaths in particular for all anticoagulants (with the exception of edoxaban), is more than sufficient to conduct the index analyses. Indeed, edoxaban is the latest addition among the approved NOACs, with the lowest numbers of FAERS records, therefore exhibiting large CIs. Nevertheless, even these relatively scarce edoxaban data are consistent with rivaroxaban and apixaban death co-reporting. The double, and even triple digits of $\chi^{2}$ estimates suggest the validity of the index dataset. Second, the quantity of adverse reports composition is reflecting the current clinical use of NOACs when warfarin is still dominant around the world. This pattern is important, suggesting a strong match between FAERS reports and 'real-life' clinical scenarios. Third, the FAERS management should be acknowledged for eliminating brand names from the reports, making the database easy to explore and navigate. Fourth, in FAERS, the $\mathrm{p}$ values for death risks between drugs are high, for example, the $\mathrm{p}$ difference in death signal between NOACs combined and warfarin is as much as $6.05 \mathrm{e}^{-17}$ (see table 1 for details). In other terms, this number means that the $p$ value for significance holds 16 zeros after the comma, and the 17th-19th digits are 605 .

The manufacturers watch the FAERS data very carefully. Sometimes delay of adverse event reporting has been encountered. ${ }^{12}$ There was also an over 9 months recent gap (now fixed) in dabigatran FAERS reporting, while cases for other NOACs are consistently updated on a quarterly basis. There are no reasons to believe that FAERS reporting has been biased against dabigatran, for example, hiding unreported death cases for other anticoagulants. Indeed, some cases are probably missing from FAERS, but here the reported trends of death distribution are still consistent.

FAERS captures real-world outcomes (like death) in a large population and under conditions that may have been overlooked in controlled trials. Clinical trials usually do not have the power to detect the whole spectrum of adverse drug reactions. Spontaneous adverse reaction reports as for example available in postmarketing repositories such as FAERS allow to screen such large data volumes for safety signals, data mining algorithms based on the concept of disproportionality have been developed. Because disproportionality analysis is based on spontaneous reports submitted for a large number of drugs and adverse event types, one might consider using these data to compare safety profiles across drugs. In fact, recent publications have promoted this practice, claiming to provide guidance on treatment decisions to healthcare decision makers. However, disproportionality should be used with caution and cannot be used for comparative drug safety analysis beyond basic hypothesis generation because measures of disproportionality are often missing the incidence denominators, may be subject to severe reporting bias and not adjusted for confounding. Hypotheses generated by disproportionality analyses must be investigated by more robust methods before they can be allowed to influence clinical decisions. Nevertheless, FAERS may provide a more realistic snapshot of the effects of anticoagulants in clinical settings. However, there are many caveats and assumptions associated with our study like undetected duplicates, which we have sought to mitigate. In fact, FAERS was developed and implemented to record safety outcomes but not as a formal registry rather than a repository of adverse events. In this context, it is important to underline that 'DEATH' may be theoretically reported as an adverse event (ie, as a preferred term) or as an outcome (ie, as the final clinical event resulting from the adverse event). In the latter scenario, causality is even more challenging and questionable, because it is unfeasible to attribute the actual death to the drug, especially considering the complexity of patients. Further research should focus on the nature of death, attempting to distinguish cardiovascular from non-cardiovascular death, and establishing the frequency of death among/across the different system organ classes and indications. Also critical is whether or not bleeding reports mainly contribute to the FAERS death rates.

When it comes to the interpretation of the results, it is obviously much too early to declare that dabigatran causes more deaths than other anticoagulants, and any alarmism will be premature. Much more detailed FAERS mining is needed with regard to doses, timing and sources of dabigatran reporting, as well as potential demographic and geographical differences. The finding is particularly surprising given the opposite mortality trends observed in the large Medicare dataset of dabigatran versus warfarin. ${ }^{13}$ Similarly, but in the opposed direction, rivaroxaban in FAERS fares much better than in the Medicare cohort reported by the same group. ${ }^{14}$ The FAERS data also contradict the dabigatran death benefit observed over warfarin in a relatively large US Department of Defense database. ${ }^{15}$ 
How can registry and repository examinations deviate so profoundly as between our FAERS analysis and the recent Medicare data? ${ }^{13}$ There are fundamental differences in the methodological approaches between these two studies: (1) Graham and colleagues targeted an elderly US Medicare ( $>65$ years) population while we analysed all international fatalities co-reported with anticoagulants. (2) In contrast to the Medicare analysis which had a number of exclusion criteria (ie, shorter than 6-month Medicare enrolment, patients in nursing facilities or on dialysis which notably is a driver of mortality, kidney transplant, mitral valve disease, heart valve repair or replacement, deep vein thrombosis, pulmonary embolism, joint replacement surgery, etc), our data are all inclusive. (3) The time window of Graham et $a l^{13}$ analysis was limited to 26 months, while our work included all dabigatran and warfarin reports available in FAERS with the cut-off date 31 December 2015, hence representing more than 10.5 years of dabigatran real-world experience. (4) The Medicare extraction was submitted to extensive propensity matching comprising a lengthy list of sociodemographic factors, medical conditions, medication use at baseline and so on, to a degree which carries the risk of producing a study population that ends up being fairly balanced but detached from a real-world population. And finally and most importantly (5), the number of deaths is over 10 times higher in the present analysis than in the Medicare report: in Graham et al the number of recorded deaths was 603 for dabigatran and 744 for warfarin, respectively, while our analysis captures 6989 deaths for dabigatran and 19493 for warfarin, creating a statistical strength that is substantially higher in the current assessment.

There are important explanation models as to the astounding finding of the dabigatran-associated data. The most important stems from so-called prescriber bias, that is, the fact that physicians opted on dabigatran prescription based on a precoined perception of which population would derive most advantage from this drug as opposed to warfarin. For example, if many prescribing doctors thought that low-dose dabigatran would be a better choice in all their patients who had documented poor international normalised ratio control in their preceding warfarin treatment, then it is clear that the target population of dabigatran users would be skewed towards a poorer outcome-not only in their previous warfarin treatment but even more so for dabigatran. This is particularly likely in light of the early approval of dabigatran as first NOAC on the market. In this context, prescriber specialty may play a role: it has been shown that rivaroxaban is more often prescribed by cardiologists while dabigatran more often by family practitioners. ${ }^{14}$ The implications of such observations are unknown. Unfortunately, it will be nearly impossible to do the quality analysis based on anticoagulant dosage because dose is not uniformly reported in FAERS. Furthermore, and on top of specialty bias, population bias may play an important role in such reporting as well-the database does in no way correct for variations in the baseline characteristic of populations that have been treated with the various anticoagulants. Importantly, the index analysis of data is limited due to known bias of spontaneous reporting system. Some of non-modifiable limitations such as the inability to fully assess causality, under-reporting and lack of exposure data should be acknowledged. Indeed, misspelled or abbreviated terms may change the absolute numbers, although not biased towards dabigatran since may be attributed to all NOACs and warfarin as well. Finally, the FAERS database is completely devoid of any statistical adjustments, with covariates and confounding factors freely interfering with the recorded numbers. The time aspect of drug scrutiny is extremely important when reporting adverse reactions/deaths are to be assessed but missing from the current analyses. For example, since dabigatran was the first NOAC on the market, there were skyrocketing numbers of bleeding side effects immediately after its market introduction potentially increasing mortality. Therefore, the repeated annual death reports will be critical for our better comprehension of the index NOAC's data. While the FAERS database holds a great number of patients and records, there is a legitimate concern pertaining to the interpretation of the obtained results. The interpretation of crude death rates is often complex and requires thorough knowledge of both the data available in the monitoring system and the statistical methods applied. These data are clearly hypothesis generating, with multiple missing here variables to consider in further research. Taken together, these drawbacks warrant utmost caution in the interpretation of the individual drug results. Further research should dichotomise FAERS anticoagulant cases when NOACs/warfarin are indicated as the 'primary suspects', performing careful sensitivity tests.

There are also a few strengths to our approach. The study was conducted within a frame of a government-run uniformed international database, and we hired independent unbiased specialists for data mining and statistics, who are exclusively focusing on exploring FAERS repository. The sample size for death reports was sufficient for each anticoagulant to make reasonable comparisons, since FAERS requires mandatory death reporting. ${ }^{16}$

In conclusion, the magnitude of overall NOACs superiority over warfarin in terms of mortality is a reassuring finding. Fatalities for the NOACs show favourable trends for factor-Xa inhibitors (rivaroxiban, apixaban and endoxaban), but unfavourable signal for the direct thrombin inhibitor (dabigatran). These findings need to be interpreted with utmost caution. Obviously, these data are not sufficient to change the prescription patterns. Further analyses will be mandatory for better understanding of the NOACs mortality and safety profiles.

Acknowledgements The authors acknowledge Heart Drug Research (Wilmington, Delaware, USA) for supported FAERS data mining and statistical work. Special thanks to Dr Paul A Danese from FDAble for statistical skills and detailed FAERS mining. 
Contributors VS and VC conceived the study and planned the analytical approach. VS, SF and MHK interpreted the results. VS and SF drafted the paper. VC and MHK commented on and edited further drafts. VS produced the final manuscript.

Funding This research was supported by a grant from the Korea Health Technology R\&D Project through the Korea Health Industry Development Institute (KHIDI), funded by the Ministry of Health \& Welfare, Republic of Korea (grant number: HI14C1731) and funded by the Ministry of Education to Dr Kim.

Competing interests VS received research grants from the dabigatran, rivaroxaban and apixaban manufacturers, lecture fees from the dabigatran, apixaban and edoxaban manufacturers and consultant fees from all four NOACs manufacturers

Provenance and peer review Not commissioned; externally peer reviewed.

Open Access This is an Open Access article distributed in accordance with the Creative Commons Attribution Non Commercial (CC BY-NC 4.0) license, which permits others to distribute, remix, adapt, build upon this work non-commercially, and license their derivative works on different terms, provided the original work is properly cited and the use is non-commercial. See: http://creativecommons.org/ licenses/by-nc/4.0/

(c) Article author(s) (or their employer(s) unless otherwise stated in the text of the article) 2017. All rights reserved. No commercial use is permitted unless otherwise expressly granted.

\section{REFERENCES}

1. Connolly SJ, Ezekowitz MD, Yusuf S, et al. Dabigatran versus warfarin in patients with atrial fibrillation. $N$ Engl J Med 2009;361:1139-51.

2. Patel MR, Mahaffey KW, Garg J, et al. Rivaroxaban versus warfarin in nonvalvular atrial fibrillation. N Engl J Med 2011;365:883-91.

3. Granger CB, Alexander JH, McMurray JJ, et al. Apixaban versus warfarin in patients with atrial fibrillation. $N$ Engl J Med 2011;365:981-92.

4. Giugliano RP, Ruff CT, Braunwald E, et al. Edoxaban versus warfarin in patients with atrial fibrillation. N Engl J Med 2013;369:2093-104.

5. Ruff CT, Giugliano RP, Braunwald E, et al. Comparison of the efficacy and safety of new oral anticoagulants with warfarin in patients with atrial fibrillation: a meta-analysis of randomised trials. Lancet 2014;383:955-62.

6. Marciniak TA, Cherepanov V, Golukhova E, et al. Follow-up rates in oral antithrombotic trials. JAMA Intern Med 2016;176:257-9.

7. Marciniak TA. Antiplatelet and anticoagulant drugs: cancer risks. Application number: 206143orig1s000 Medical Review(s).Center for Drug evaluation and research. December 2014:62-3. 72-73, 76, 81,82. http://www.accessdata.fda.gov/drugsatfda_docs/nda/2015/ 2061430rig1s000MedR.pdf. (accessed 21 Oct 2016).

8. Adverse event Reporting System (AERS). http://www.fda.gov/ Drugs/GuidanceComplianceRegulatorylnformation/Surveillance/ AdverseDrugEffects/default.htm (assessed 4 Oct 2016).

9. Duggirala $\mathrm{HJ}$, Tonning JM, Smith $\mathrm{E}$, et al. Use of data mining at the food and drug administration. J Am Med Inform Assoc 2016;23:428-34

10. Böhm R, von Hehn L, Herdegen T, et al. OpenVigil FDA - Inspection of U.S. American adverse drug events pharmacovigilance data and novel clinical applications. PLoS One 2016;11:e0157753.

11. Evans SJ, Waller PC, Davis S. Use of proportional reporting ratios (PRRs) for signal generation from spontaneous adverse drug reaction reports. Pharmacoepidemiol Drug Saf 2001;10:483-6.

12. Heavey S. FDA warns Pfizer for not reporting side effects. http:// www.reuters.com/article/us-pfizer-fda-idUSTRE6586PE20100610 (assessed 19 Oct 2016).

13. Graham DJ, Reichman ME, Wernecke M, et al. Cardiovascular, bleeding, and mortality risks in elderly medicare patients treated with dabigatran or warfarin for nonvalvular atrial fibrillation. Circulation 2015;131:157-64.

14. Graham DJ, Reichman ME, Wernecke M, et al. Stroke, bleeding, and mortality risks in elderly Medicare beneficiaries treated with dabigatran or rivaroxaban for nonvalvular atrial fibrillation. JAMA Intern Med 2016;176:1662.

15. Villines TC, Schnee J, Fraeman K, et al. A comparison of the safety and effectiveness of dabigatran and warfarin in non-valvular atria fibrillation patients in a large healthcare system. Thromb Haemost 2015;114:1290-8.

16. Expedited safety Reporting requirements for human drug and biological products. http://www.fda.gov/Safety/MedWatch/ Safetylnformation/Safety-RelatedDrugLabelingChanges/ucm120262. htm (assessed 19 Oct 2016). 